\title{
Descartes' Grundgedanken in medizinhistorischer Sicht
}

\author{
Von René Bernoulli
}

Es ist in geistesgeschichtlichen Werken nahezu Tradition, René Descartes (Renatus Cartesius, 1596-1650) an den Beginn des Denkens der Neuzeit zu stellen. Man sieht in ihm den Hauptbegründer der modernen Erkenntniskritik; sein Einfluß läßt sich bis in die Gegenwart aufzeigen. Anders als die von der «objektiven Welt» ausgehende Scholastik des Mittelalters stellt Descartes den methodischen Zweifel an den Anfang seines Philosophierens - «de omnibus dubitandum» ist der Ausgangspunkt des Cartesianismus ${ }^{1}$. Das «cogito, ergo sum » ${ }^{2}$ soll anschließend das durch die radikale Skepsis ausgelöste Unbehagen, dieses anthropologisch unbefriedigende Gefühl des Mißmutes über den Zweifel am je eigenen Sein, beseitigen.

Bei aller Wertschätzung, welcher sich die Cartesische Philosophie erfreut, darf indes nicht übersehen werden, daß ihre Theoreme nicht uneingeschränkte Anerkennung fanden. So sah Descartes sich schon frühzeitig mit seinem Zeitgenossen, dem Abbé Pierre Gassend (1592-1655), als Mathematiker und Philosoph bekannter unter dem Namen Gassendi, in Diskussionen verwickelt ${ }^{3}$. Es gab immer wieder große Denker, die sich kritisch über den Cartesianismus, in erster Linie das «cogito, ergo sum », geäußert haben ${ }^{4}$. Besonders heftige Angriffe führte Friedrich Nietzsche (1844-1900), der Descartes als «oberflächlich» taxiert ${ }^{5}$, selbst wenn dieser «hundert Mal in Rechtschaffenheit den ersten Deutschen überlegen ${ }^{6}$ sein soll. Aber auch in der Gegenwart ist die Auseinandersetzung mit Descartes nicht verstummt. Es sei beispielsweise der zur Medizin- wie zur Philosophiegeschichte gehörende Karl Jaspers (1883-1969) zitiert: «Aber auch wir gehören bei aller Bewunderung für seine historische Größe zu denen, die an dem Gehalt und an der Methode seiner Philosophie als einer ewigen Gestalt philosophischer Wahrheit zweifeln [...]. Wenn wir dann beobachten, wie Descartes' Denken in anderen Köpfen wirkte, so fragen auch wir, ob er durch seine Größe nicht zugleich eine Macht war, die das Philosophieren aller, die ihm folgten, zwar entzündete, aber zugleich auf einen Abweg brachte, nicht nur durch seine Methode, sondern auch durch seine Inhalte. ${ }^{7}$

Doch nun soll, was die Descartes-Kritik angeht, Descartes selbst zu Worte kommen: Es gehört zweifellos zu den merkwürdigsten Besonderheiten der Geistesgeschichte, daß zu den schärfsten Kritikern des Cartesianismus sein Urheber selbst zählt. Nämlich: Descartes war nicht wenig stolz auf seine medizinischen 
Kenntnisse. So schreibt er am 9. Januar 1639 seinem gelehrten Freund, Pater Marin Mersenne (1588-1648), noch etwas bescheiden: «i'ay acquis quelque peu de connoissance de la medecine. ${ }^{8}$ Im Brief vom 20. Februar 1639, ebenfalls an Mersenne gerichtet, deutlicher und selbstbewußter: «En effet, i'ay consideré non seulement ce que Vezalius \& les autres écriuent de l'Anatomie, mais aussi plusieurs choses plus particulieres que celles qu'ils écriuent, lesquelles i'ai remarquées en faisant moy-mesme la dissection de diuers animaux. C'est vn exercice où ie me suis souuent occupé depuis vnze ans, \& ie croy, qu'il n’y a gueres de Medecin qui y ait regardé de si prés que moy. ${ }^{9}$

Nach dem letzten Zitat kannte Descartes Vesals De humani corporis fabrica libri VII, was nicht überrascht. Hingegen fällt auf, daß Descartes sich bezüglich seiner anatomischen Ansichten auf eigene Tiersektionen beruft, was gegenüber Vesal anatomiehistorisch einen prinzipiellen Rückschritt bedeutet ${ }^{10}$. Aber auch in der Physiologie ging Descartes selbständige Wege. Er kannte jedenfalls das 1628 erschienene grundlegende Werk von William Harvey, De motu cordis et sanguinis $^{11}$. Auf Grund eigener Untersuchungen und U̇berlegungen entwickelte er jedoch in bezug auf die Motorik der Herztätigkeit und des Blutkreislaufes eigene, den Ergebnissen von Harvey nur teilweise entsprechende Theorien. In seinem vom 9. Februar 1639 datierten Schreiben an P. Mersenne findet sich zu dieser Thematik ein besonders aufschlußreicher Abschnitt:

"Vous me mandez qu'vn Medecin Italien [Parisanus Venetus] a escrit contre Herueus de motu cordis, \& que cela vous fait estre marris de ce que ie me suis engagé a escrire de cette matiere; en quoy ie vous diray franchement que ie ne vous sçaurois remercier de vostre charité en mon endroit: car il faut que vous ayez bien mauuaise opinion de moy, puis que, de cela seul qu'on vous dit qu'vn autre a escrit, non pas contre moy: (car, bien que ceux qui ne regardent que l'ecorse iugent que i'ay escrit le mesme qu'Herueus, a cause de la circulation du sang, qui leur donne seule dans la veue, i'explique toutefois tout ce qui appartient au mouuement du cœur d'vne façon entierement contraire a la siene); mais de ce que quelqu'vn a escrit quelque chose, que vous imaginez estre contre moy, sans auoir oui ses raisons, ny mesme sçauoir s'il est habile homme, vous supposez incontinent que i'ay failly. Ie voy de là, \& de plusieurs autres telles choses, que les bonnes raisons ont fort peu de force pour persuader la verité, ce qui me fait presque resoudre d'oublier tout à fait a escrire, \& n'estudier iamais plus que pour moy mesme. Cependant ie veux bien qu'on pense que, si ce que i'ay escrit de cela, ou des refractions, ou de quelque autre matiere que i'aye traitée en plus de 3 lignes dans ce que i'ay fait imprimer, se trouue faux, tout le reste de ma Philosophie ne vaut rien. ${ }^{12}$ 
Es ist eine philosophie- wie medizingeschichtlich gleicherweise merkwürdige Tatsache, daß Descartes selbst seine Philosophie de facto in Frage stellt, und zwar auf Grund seiner medizinischen Theorien. Er erklärt jedenfalls die Medizin zu einer der Philosophie übergeordneten Instanz, eine geistesgeschichtlich gewiß bemerkenswerte Tatsache. Man mag auf den ersten Blick diese von Descartes angeführten Bezüge zwischen Medizin und Philosophie als fragwürdig empfinden. Doch ist diesbezüglich zu bemerken, daß rund anderthalb Jahrhunderte später kein Geringerer als Immanuel Kant (1724-1804) in seinem Spätwerk, Der Streit der Fakultäten, die medizinische «Fakultät [...] der philosophischen sehr nahe verwandt» sieht ${ }^{13}$. Die Geistesgeschichte zeigt übrigens zur Genüge, in welchem Maße beide Disziplinen im Laufe der Jahrhunderte oft miteinander verwoben waren. Letztlich geht es beiden um die anthropologische Zentralfrage «Was ist der Mensch ?», ${ }^{14}$ es sei denn, man erkläre die eine als rein handwerkliche, die andere als gedankliche «Technè», die nach sturen Regeln zu betreiben wäre. Es ist daher an sich kein absurdes Vorgehen, in der Medizin einen Censor der Philosophie zu sehen - Medicina iudex Philosophiae.

Was nun Descartes im besonderen angeht, so mißt er nach der eben zitierten Briefstelle an seinen eigenen medizinischen Thesen Richtigkeit und Wahrheitsgehalt seiner Philosopheme. Bei seiner Beweisführung scheint er sich freilich die Frage nicht vorgelegt zu haben, ob die Prämisse seines Syllogismus hieb- und stichfest sei. Sie ist es nicht; Descartes ist einem "prōton pseudos », einer «ersten Lüge», zum Opfer gefallen: Denn es steht jedenfalls fest, daß viele seiner biologischen Ansichten irrig sind ${ }^{15}$. Die in der «Cinquiesme partie», S. $41 \mathrm{ff}$, des 1637 von ihm selbst veröffentlichten Discours de la Méthode ${ }^{16}$ dargestellten Theorien über Herztätigkeit und Blutkreislauf sind bekanntlich zu einem großen Teil falsch. Und ebensowenig entsprechen zahlreiche, im postum erschienenen Traité de l'Homme ${ }^{17}$ aufgestellte Hypothesen den Tatsachen, wie z.B. das angeblich vom Corpus pineale - nach Descartes Sitz des Intellekts ${ }^{18}$ - beherrschte ${ }^{19}$ «Gebläse $»,{ }^{20}$ das in den Nervensträngen stattfinden und ihre Funktion ausmachen soll $^{21}$. Somit hat, scharf formuliert, Descartes selbst, und zwar auf Grund eigener Prämissen und Kriterien, das Urteil über seine ganze Philosophie gefällt - «tout le reste de ma Philosophie ne vaut rien»!

Angesichts der von Descartes eigenhändig ins Feld geführten Beziehungen zwischen seiner Philosophie einerseits und seiner Anatomie und Physiologie anderseits ist allerdings zu berücksichtigen, daß - so Karl Jaspers - es keine Philosophie gibt, «in deren Gedankengängen nicht Widersprüche aufgezeigt wären ». ${ }^{22}$ Descartes' Selbstkritik gehört sicher in dieses Kapitel philosophischer Existenz. Anders verhält es sich aber mit dem Ansatzpunkt seiner Philosophie, ihrem Ur- 
sprung, wenn man will. Descartes geht vom methodischen und grundsätzlichen Zweifel aus, dessen Grundzüge er in der «Quatriesme partie», S. 32 f., des Discours de la méthode niedergelegt hatte:

«Ie ne sçay si ie doy vous entretenir des premieres meditations que i’y ay faites, car elles sont si Metaphysiques \& si peu communes, qu'elles ne seront peutestre pas au goust de tout le monde: Et toutefois affin qu'on puisse iuger si les fondemens que i'ay pris sont assez fermes, ie me trouue en quelque façon contraint d'en parler. I'auois dés long temps remarqué que pour les meurs il est besoin quelquefois de suiure des opinions qu'on sçait estre fort incertaines, tout de mesme que si elles estoient indubitables, ainsi qu'il a esté dit cy dessus: mais pourcequ'alors ie desirois vacquer seulement a la recherche de la verité, ie pensay qu'il faloit que ie fisse tout le contraire, \& que ie reiettasse comme absolument faux tout ce en quoy ie pourrois imaginer le moindre doute, affin de voir s'il ne resteroit point aprés cela quelque chose en ma creance qui fust entierement indubitable. Ainsi a cause que nos sens nous trompent quelquefois, ie voulû supposer qu'il n'y auoit aucune chose qui fust telles qu'ils nous la font imaginer: Et pourcequ'il y a des hommes qui se méprenent en raisonnant, mesme touchant les plus simples matieres de Geometrie, \& y font des Paralogismes, iugeant que i'estoit suiet a faillir autant qu'aucun autre, ie reiettay comme fausses toutes les raisons que i'auois prises auparauant pour Demonstrations: Et enfin considerant que toutes les mesmes pensées que nous auons estant esueillez, nous peuuent aussy venir quand nous dormons sans qu'il y en ait aucune pour lors qui soit vraye, ie me resolu de feindre que toutes les choses qui m'estoient iamais entrées en l'esprit n'estoient non plus vrayes que les illusions $l^{23}$ mes songes. »

Das In-Frage-Stellen einer jeden Gewißheit, wie sie in diesem Zitat unumwunden zum Ausdruck kommt, ist eine der Stellen, auf denen Descartes' Bedeutung für die Zukunft beruht. Seine Antwort auf diesen Zweifel ist bekannt: «ie pense, donc ie suis», wie die Urform lautet ${ }^{24}$.

Für jetzt soll indes nur der Cartesische methodische Zweifel weiter erörtert werden. Descartes' Skepsis ist nicht, wie das letzte Zitat möglicherweise den Anschein erweckt, eine ursprüngliche, einzig dem eigenen Denken entsprungene Schöpfung. Bereits in der Antike hatten ja andere schon den (philosophischen) Zweifel propagiert und diskutiert, wobei vornehmlich auf Sextus Empiricus hinzuweisen ist. Dieser um $200 \mathrm{n}$. Chr. in Alexandria und Athen lebende griechische Arzt hinterließ in seinen Werken (Pyrrhonische Skizzen, Gegen die Mathematiker, Gegen die Dogmatiker) eine wahre Summe, die noch heute als wichtigste Quelle zur Kenntnis des antiken Skeptizismus, nach ihrem größten Vertreter Pyrrhon aus Elis (um 360-270 v. Chr.) auch Pyrrhonismus genannt, gilt. 
Wenn es auch möglich ist, daß Descartes die Bücher des Sextus Empiricus kannte ${ }^{25}$, so muß doch, wenn bei ihm vom Zweifel die Rede ist, in erster Linie an Michel Eyquem, Seigneur de Montaigne (1533-1592), gedacht werden. Dieser gemeinhin unter der Kurzform «Montaigne» zitierte Anthropologe hatte in seinen zu einem "Weltbuch ${ }^{26}$ gewordenen Essais, die ob ihrer Aktualität ${ }^{27}$ immer wieder ediert werden, den Zweifel als Methode in einem nicht zu überbietenden Radikalismus ausgearbeitet ${ }^{28}$. Eine vergleichende Lektüre von Descartes' philosophischen Werken und den Essais führt unweigerlich zur Einsicht, daß Descartes sich intensiv mit Montaigne beschäftigt hatte; zwischen den zwei Denkern bestehen geistesgeschichtlich fortwirkende Bezüge. Es kann daher nicht überraschen, wenn z. B. Etienne Gilsons Ausgabe des Discours de la méthode ( ${ }^{3}$ Paris 1962) in ihrem kritischen Anmerkungsapparat zahlreiche Hinweise auf Montaigne enthält, was der Feststellung eines anderen modernen Philosophen - Léon Brunschvicg - entspricht: «Descartes entreprend de répondre à Montaigne, ses conclusions lui donnent tort sur tous les points; mais c'est après s'être mis à son école, après avoir tout accepté de lui. ${ }^{29}$ An der Tatsache, daß Descartes Montaignes Essais kannte, kann nicht gezweifelt werden. So erwähnt er in seinem philosophisch wichtigen, vom 23. November 1646 datierten Schreiben an den Marquis von Newcastle den Autor der Essais mehrmals mit Namen ${ }^{30}$.

Descartes zählt zu den großen Lesern Montaignes ${ }^{31}$. Aus Montaignes Essais entnahm er den radikalen methodischen $Z_{w}$ eifel $^{32}$; von diesem ausgehend, entwickelte er ein eigenes philosophisches System, das von größtem Einfluß auf das Reflektieren kommender Generationen sein sollte. «Il était réservé à Descartes de faire une révolution dans les sciences. Il avait appris à douter, pour détromper avant d'instruire. Son doute méthodique fut le plus grand instrument qu'on pût rendre à l'esprit humain. ${ }^{33}$

Dieser auf Montaigne zurückgehende methodische Zweifel wird sich nicht nur in der Entwicklung der Reflektierweise, sondern auch in der Evolution der Medizin wirksam erweisen. Was denn anders als Zweifel am Überlieferten treibt etwa einen Claude Bernard (1813-1878) dazu, die verifizierbare Untersuchung an den Beginn einer jeden exakten Forschung zu stellen ${ }^{34}$, vornehmlich aber das reproduzierbare Experiment an den Anfang der Physiologie ${ }^{35}$ ? «Le douteur est le vrai savant. ${ }^{36}$

Nun geht aber in einer Art dialektischer Bewegung dieser grundsätzliche Zweifel in ganz besonderem Maße auf biologische Gegebenheiten zurück. Schon Sextus Empiricus berief sich in seinen Pyrrhonischen Skizzen auf medizinische und naturwissenschaftliche Beobachtungen ${ }^{37}$. Montaigne wird in seinem zwölften Kapitel des zweiten Buches der Essais, in der «Apologie de Raimond Sebond ${ }^{38}$ diese 
Argumente übernehmen, sie weiter ausbauen und zu einem der Pfeiler seiner anthropologisch orientierten Philosophie machen. Schon diese Bezüge rechtfertigen es, in Michel de Montaigne eine die Medizingeschichte interessierende Gestalt zu sehen.

Es ergibt sich: Durch den Descartes zugeschriebenen Einfluß auf die Evolution der Reflektierweise wurden die Verdienste seiner Vorgänger in vielerlei Hinsicht vergessen oder übersehen. Die chronologische Einordnung und geistesgeschichtliche Richtigstellung des Cartesischen Grundgedankens, des radikalen Zweifels, mindert Descartes' Bedeutung sicher nicht, am allerwenigsten seine Pionierleistung als Mathematiker. Andererseits zeigt Descartes, daß auch beim hervorragenden Denker «die Unabhängigkeit des Philosophen im Menschen falsch wird, wenn ein Stolz darin liegt », ${ }^{39} \mathrm{kurz}$, daß auch das Genie der «condition humaine», wie Montaigne die menschliche Grundbefindlichkeit bezeichnet, unterworfen ist.

\section{Anmerkungen}

$1 \mathrm{Zu}$ den hier verwendeten philosophischen Termini vgl. u. a. in Johannes Hoffmeister, Wörterbuch der philosophischen Begriffe, 2. Aufl., Hamburg [1955], die Stichworte «Cartesianismus», S.133f., "cogito, ergo sum», S.14.1f., und "de omnibus dubitandum», S. 160 .

2 Dieser «formulierte Grundsatz» der Cartesischen Metaphysik und «Hauptsatz der neueren abendländischen Philosophie überhaupt» (Johannes Hoffmeister, a.a. O., S. 141) soll in der vorliegenden Studie nicht weiter erörtert werden.

${ }^{3}$ Vgl. hierzu das postum erschienene Werk: PETRI GASSENDI / DISQVISITIO / METAPHYSICA. / Seu / DVBITATIONES, / ET INSTANTIAE : / Adversus / RENATI CARTESII / Metaphysicam, \& Responsa. / [Titelvignette] / AMSTERODAMI, Apud IOHANNEM BLAEV, / M DCXLIV. - Aber schon vor dieser 337 Seiten umfassenden Publikation, noch zu Lebzeiten Descartes' und Gassendis, hatte Descartes Gassendis «dubitationes et instantias» und seine eigenen diesbezüglichen «responsa» in seinem großen Werk, Meditationes de prima philosophia, ${ }^{1}$ Paris 1641, veröffentlicht (S. 355 bis $551)$.

${ }^{4}$ Siehe hierzu die von Johannes Hoffmeister gegebene summarische Übersicht, a.a.O., S.141f.

${ }^{5}$ Friedrich Nietzsche, Jenseits von Gut und Böse, 5. Hauptstück, 191.

6 Id., Ecce homo, «Der Fall Wagner», 3.

7 Karl Jaspers, Descartes und die Philosophie, 4., unveränderte Aufl., Berlin 1966, S. 5 f.

8 Euvres de Descartes, publiées par Charles Adam \& Paul Tannery, Correspondance II, Paris 1898, S. 480. - Wir geben hier wie in allen folgenden Zitaten den Originaltext einschließlich allfälliger Druckfehler buchstabengetreu wieder.

9 A.a.O., S. 525. 
10 Ob Descartes sich später an Sektionen an der menschlichen Leiche beteiligte, soll hier nicht weiter untersucht werden. Er wich jedenfalls nie von seinen angeblich durch Tiersektionen gewonnenen Ansichten ab.

11 So findet sich z.B. schon in der Urausgabe von 1637 des Discours de la Méthode (genauer Titel s. unten Anm.16), in der "Cinquiesme partie», S. 51 die Marginalie: «Heruaeus de motu cordis.» - Auf Grund eingehender Konfrontation des Urtextes mit modernen Editionen scheint es mir ratsam, auf Descartes' authentischen Text buchstabengetreu zurückzugreifen. Es ist mir ein Bedürfnis, den Herren Konservatoren der Bibliothèque Nationale in Paris meinen Dank auszusprechen für die Bereitwilligkeit, mit der sie mir die seltenen Werke zur Verfügung stellten.

12 Euvres de Descartes, Correspondance II, a.a. O., S. $500 \mathrm{f}$.

13 Immanuel Kant, Der Streit der Fakultäten, «Erster Abschnitt, I, 1, C. Eigentümlichkeit der medizinischen Fakultät».

${ }^{14}$ So in Immanuel Kant's Logik / Ein Handbuch zu Vorlesungen, «Einleitung, III. Begriff von der Philosophie überhaupt», und in Kants Brief an C. Fr. Stäudlin vom 4. Mai 1793.

15 Siehe hierzu bes. Louis Chauvois, Descartes, sa méthode et ses erreurs en physiologie, Paris 1966.

${ }^{16}$ Der genaue Titel dieses für den Cartesianismus grundlegenden, zunächst anonym erschienenen Werkes lautet: «DISCOURS / DE LA METHODE / Pour bien conduire sa raison, \& chercher / la verité dans les sciences. / PLUS / LA DIOPTRIQVE. / LES METEORES. / ET / LA GEOMETRIE. / Qui sont des essais de cete METHODE. / [Titelvignette] / A LEYDE / De l'Imprimerie de IAN MAIRE. / M D C XXXVII. / Auec Priuilege.»-Eine Reproduktion des Titelblattes findet sich in der von Lüder Gäbe besorgten französisch-deutschen Edition des Discours, Philosophische Bibliothek Bd. 261, Nachdruck 1964.

17 In Anbetracht der Bedeutung, die diesem Werk für die Zukunft zukommt, soll die Geschichte seiner Veröffentlichung kurz dargestellt werden: Descartes selbst hatte seine Arbeit nicht herausgegeben. Die erste Edition erschien erst postum unter dem Titel: «RENATUS DES CARTES / DE / HOMINE / FIGVRIS / ET / LATINITATE DONATUS / A / FLORENTIO SCHUYL, / Inclytae Urbis Sylvae Ducis Senatore, \& ibidem / Philosophiae Professore. / [Titelvignette] / LVGDVNI BATAVORVM, / Apud PETRVM LEFFEN \& FRANCISCVM / MOYARDVM. / M D CLXII. (Ein anderer Abzug gibt als Verleger: «Apud FRANGISCVM MOYARDVM / \& PETRVM LEFFEN. ») Nach diesen Angaben hatte der in Herzogenbusch ('s Hertogenbosch, kurz den Bosch, Hauptstadt der niederländischen Provinz Nordbrabant) lebende Arzt, Botaniker, Magistrat und Philosophieprofessor Florentinus Schuyl (1633-1670) Descartes' Text ins Lateinische übertragen. Zwei Jahre später kam die Arbeit in der französischen Originalsprache heraus unter Beifügung des ausführlichen Kommentars von Ludovicus de La Forge (auch Delaforge), eines um die Mitte des 17.Jahrhunderts lebenden Arztes und cartesischen Philosophen: «L'HOMME / DE RENÉ / DESCARTES / ET VN TRAITTÉ / DE LA FORMATION DV FOETVS / DV MESME AVTHEVR. / Auec les Remarques de LOVYS DE LA FORGE, / Docteur en Medecine, demeurant à la Fleche, / Sur le Traitté de l'Homme de RENÉ DESCARTES, / \& sur les Figures par luy inuentées. / [Titelvignette] / A PARIS, / Chez CHARLES ANGOT, Libraire Iuré, ruë / S. Iacques, au Lion d'Or. / M. DC. LXIV. / AVEC PRIVILEGE DV ROY.» Das Werk hat fol- 
gende Gliederung: 5 nicht numerierte Seiten, die Widmung an «Monseigneur de Colbert» enthaltend. - 62 nicht numerierte Seiten «Préface», auf letzter Seite ebenfalls das königliche Druckprivileg. - S.1-107 die eigentliche Abhandlung, «L'HOMME / DE RENÉ / DESCARTES». - S. 109-170 «LA DESCRIPTION / DV CORPS HVMAIN, / ET DE TOVTES SES FONCTIONS; / Tant de celles qui ne dependent point de l'Ame,/ Que de celles qui en dependent. / Et aussi la principale cause de la formation de ses membres.» Auf den Textseiten dieses Abschnittes findet sich jedoch jeweils über dem eigentlichen Text die Angabe "DE LA FORMATION DV FOETVS», nach der diese Studie gemeinhin benannt wird. - S.171-4.08 «REMARQVES / DE LOVIS DE LA FORGE / DOCTEVR EN MEDECINE, / Sur le Traitté de l'Homme, DE RENÉ DESCARTES: / Et sur les figures par luy inuentées.»-S.409-448 die von La Forge besorgte französische Übersetzung des lateinischen Vorwortes von Florentinus Schuyl der Ausgabe von 1662. - Anschließend 8 nicht numerierte Seiten mit der Inhaltsangabe. - Ein besonderes Problem bilden die Abbildungen. Nach den Titeln beanspruchen sowohl Schuyl wie de La Forge, eigene Bilder beigefügt zu haben. Leider ist das authentische Manuskript verlorengegangen; Kopien davon gingen noch zu Lebzeiten Descartes' durch verschiedene Hände, vgl. Descartes' Brief an P. Mersenne vom 23. November 1646. Unsere Zitate aus dem Traité de l'Homme sind ausnahmslos der Edition des Jahres 1664 entnommen.

18 "In ihr [der Glandula pinealis] suchte Cartesius den Sitz der Seele, - fand ihn aber nicht», bemerkt Joseph Hyrtl vielleicht etwas sarkastisch (Lehrbuch der Anatomie des Menschen, 20. Auflage, Wien 1889, S. 904). Doch soll Descartes selbst in seinem Traité de l'Homme, a.a.O., S. 73, zu Worte kommen: «Or entre ces figures, ce ne sont pas celles qui s'impriment dans les organes des sens exterieurs, ou dans la superficie interieure du cerueau, mais seulement celles qui se tracent dans les esprits sur la superficie de la glande $\mathrm{H}$, où est le siege de l'Imagination, \& du sens commun, qui doiuent estre prises pour les idées, c'est à dire pour les formes ou images que l'Ame Raisonnable considerera immediatement, lors qu'estant vnie à cette machine elle imaginera ou sentira quelque objet.» - Unter der "glande $\mathbf{H}$ » ist immer das Corpus pineale zu verstehen, wie aus den beigefügten Abbildungen hervorgeht. - Schrägdruck im Original.

19 A.a.O., S. $87 \mathrm{f}$.: «Considerez aussi que les seuls mouuemens de la glande $H$, sont suffisans pour changer la situation de ces tuyaux, \& en suitte toute la disposition du corps de l'œil, ainsi qu'il a tantost esté dit en general qu'ils peuuent faire mouuoir tous les membres. »

20 A.a. O., S. 57 f.: «Si vous auez iamais eu la curiosité de voir de prés les orgues de nos Eglises, vous sçauez comment les soufflets y poussent l'air en certains receptacles, qui ce me semble sont nommez à cette occasion les porte-vents, \& comment cet air entre de là dans les tuyaux, tantost dans les vns, tantost dans les autres, selon les diuerses façons que l'organiste remuë ses doigts sur le clauier. Or vous pouvez icy conceuoir que le cœur \& les arteres, qui poussent les esprits animaux dans les concauitez du cerueau de nostre machine, sont comme les soufflets de ces orgues, qui poussent l'air dans les portevents; \& que les objets exterieurs, qui, selon les nerfs qu'ils remüent, font que les esprits contenus dans ces concauitez entrent de là dans quelques-vns de ces pores, sont comme les doigts de l'organiste, qui, selon les touches qu'ils pressent, font que l'air entre des porte-vents dans quelques tuyaux.». 
21 A.a.0., S. 15: «Voyez donc icy par exemple le nerf A, dont la peau exterieure est comme vn grand tuyau, qui contient plusieurs autres petits tuyaux b, c, k, l, \&c. composez d'vne peau interieure plus déliée ; \& ces deux peaux sont continuës auec les deux K, L, qui enuelopent le cerueau $M, N$, o. Voyez aussi qu'en chacun de ces petits tuyaux, il y a comme vne moëlle, composée de plusieurs filets fort déliez, qui viennent de la propre substance du cerueau $\mathrm{N}, \&$ dont les extremitez finissent d'vn costé à sa superficie intérieure qui regarde ses concauitez, \& de l'autre aux peaux \& aux chairs contre lesquelles le tuyau qui les contient se termine. Mais pource que cette moëlle ne sert point au mouuement des membres, il me suffit pour maintenant que vous sçachiez qu'elle ne remplit pas tellement les petits tuyaux qui la contiennent, que les esprits animaux n'y trouuent encore assez de place, pour couler facilement du cerueau dans les muscles, où ces petits tuyaux, qui doivent icy estre comptez pour autant de petits nerfs, se vont rendre.» Dabei steht eine eingefügte Abbildung, auf die im Text verwiesen wird.

${ }^{22}$ Karl Jaspers' Vorwurf an die Philosophie wiegt schwer: «Der Widerspruch ist Kennzeichen der Unrichtigkeit. Er läßt keine Ruhe. Er muß aufgelöst werden. Aber es gibt keine Philosophie, in deren Gedankenfiguren nicht Widersprüche aufgezeigt wären» (Die großen Philosophen, München [1959], S. 86).

23 «les illusions le mes songes», Druckfehler, statt: «les illusions de mes songes».

${ }^{24}$ Discours de la méthode, a.a.O., S. 33. Im Original durch Schrägdruck hervorgehoben.

${ }^{25}$ Die Werke des Sextus Empiricus erschienen schon im 16. Jahrhundert im Druck.

26 «Die Essais sind ein Weltbuch geworden» (Hugo Friedrich, Montaigne, 2., neubearbeitete Auflage, Bern und München [1967], S. 9).

27 Vgl. hierzu beispielsweise Pierre d'Espezel, Présence et actualité de Montaigne, Paris [1957].

${ }^{28}$ Vgl. diesbezüglich z. B. meine Untersuchung «Que sais-je? Contribution à l'étude de la gnoséologie de Montaigne I», Bulletin de la Sociêté des Amis de Montaigne, $5^{\mathrm{e}}$ série, $\mathrm{n}^{\mathrm{o}} 16$, 1975 , S. 15-36.

${ }^{29}$ Léon Brunschvicg, Descartes et Pascal Lecteurs de Montaigne, Neuchâtel 1945, S. 97.

30 Dem damaligen Brauch gemäß schrieb Descartes «Montagne», was aber «Montaigne» ausgesprochen wurde.

31 «La fortune a donné à Montaigne les lecteurs les plus assidus et les plus fervents qu'auteur ait pu souhaiter: RENÉ DESCARTES et BLAISE PASCAL» (Léon Brunschvicg, a.a.O., S. 9).

32 Die Frage, welches wohl die Gründe sind, daß die zwischen Montaigne und Descartes bestehenden Bezüge im allgemeinen wenig bekannt sind, mag Léon Brunschvicg beantworten (a.a.O., S. 130): «La plupart des commentateurs de Descartes ont été des philosophes de profession qui n'ont guère lu que par distraction les Essais de Montaigne; ceux de Montaigne ont été le plus souvent des hommes de lettres qui ont reculé devant la méditation des Essais scientifiques de Descartes. C'est pourquoi n'ont pas toujours été suffisamment mis en relief les rapports de solidarité tout à la fois et d'opposition qui nous ont paru exister entre les deux œuvres.»

${ }^{33}$ Etienne Tourtelle, Histoire philosophique de la Médecine depuis son origine jusqu'au commencement du $18^{e}$ siècle, tome second, Paris, an XII (1804), S. 382. - E. Tourtelle war Professor der Medizin, zuerst in Besançon, dann in Strasbourg.

34 Claude Bernard, Introduction à l'étude de la médecine expérimentale, Paris 1865 [Uraus- 
gabe], II. Kapitel, §6, S. 85 f.: «Je résumerai le paragraphe précédent en disant qu'il me semble n'y avoir qu'une seule forme de raisonnement : la déduction par syllogisme. Notre esprit, quand il le voudrait, ne pourrait pas raisonner autrement [...]. Mais pour trouver la vérité scientifique, il importe peu au fond de savoir comment notre esprit raisonne; il suffit de le laisser raisonner naturellement, et dans ce cas il partira toujours d'un principe pour arriver à une conclusion $[\ldots]$. Ce précepte général, qui est une des bases de la méthode expérimentale, c'est le doute.»

35 Id., a. a. O., S. $90 \mathrm{f}$ : : «Quand Descartes [Hinweis auf Descartes' Discours de la méthode] part du doute universel et répudie l'autorité, il donne des préceptes bien plus pratiques pour l'expérimentateur que ceux que donne Bacon pour l'induction. Nous avons vu, en effet, que c'est le doute seul qui provoque l'expérience [ ... ]. Toutefois, quand il s'agit de la médecine et des sciences physiologiques, il importe de bien déterminer sur quel point doit porter le doute.»

${ }^{36}$ Id., a.a. O., S. 91.

37 Vornehmlich im 1. Buch, 14. Kapitel.

${ }^{38}$ Der tiefere Anlaß zu diesem größten Kapitel der Essais lieferte das Werk Liber creaturarum sive de homine, später auch Theologia naturalis genannt, des im ersten Drittel des 15. Jahrhunderts in Toulouse dozierenden Arztes, Philosophen und Theologen Raimundus Sebundus. Dieser auch Sibiude und ähnlich genannte Autor ist in der Medizingeschichte wenig bekannt.

${ }^{39}$ Karl Jaspers, Kleine Schule des philosophischen Denkens, München [1965], S.171.

\section{Summary}

Although the reasoning of Descartes had a great influence on modern philosophy, his method of thinking was disapproved by some. Strange to say Descartes himself jeopardized his own theories by emphasizing his medical views which were mostly wrong. As to the origin of his reflection - methodical and thorough-going doubt - we must put stress on the fact that this doubt had already been formulated by Michel de Montaigne in the sixteenth century in his Essais in a manner that hardly could be surpassed.

Dr. med. et phil. René Bernoulli

Amselstraße 35

4059 Basel 